1. Yilmaz A, Akkaya E, Damadoglu E, Gungor S. Occult bronquial foreing body aspiration in adults: analysis of four cases. Respirology 2004; 9: 561-3.

2. Zubairi AB, Haque AS, Husain SJ, Khan JA. Foreign body aspiration in adults. Singapore Med J 2006; 47: 415-8.

3. Limper AH, Prakash UBS. Tracheobronchial foreign bodies in adults. Ann Intern Med 1990; 112: 604-9.

4. Chen CH, Lai CL, Tsai TT, Lee YC, Perng RP. Foreign body aspiration into the lower airway in chinese adults. Chest 1997; 112: 129-33.

5. Baharloo F, Veyckermans F, Francis C, Biettlot MP, Rodenstein DO. Tracheobronchial Foreign Bodies: presentation and management in children and adults. Chest 1999; 115: 1357-62.

6. Nalaboff KM, Solis JL, Simon D. Endobronquial foreign body extraction: a new interventional approach. Chest 2001; 120: 1402-05.

7. Wain JC, Mark EJ. Case 33-1997. A 75-Year-Old Man with chest pain, hemoptysis, and pulmonary lesion. N Engl J Med 1997; 337: 1220-6.

\section{Análisis de los 7 primeros años de un programa de trasplante hepático}

\section{Sr. Director:}

Aunque con un cierto retraso con respecto a los primeros trasplantes hepáticos que se llevaron a cabo en EE.UU. durante la década de los años 60 (1), existe en España un número creciente de pacientes trasplantados desde la implantación de programas de trasplante hepático en los hospitales.

Con el propósito de conocer la actividad asistencial de los trasplantes hepáticos $(\mathrm{TH})$ en nuestro hospital, se realiza este análisis de los pacientes que reciben un TH desde el inicio dicho programa en 1998 hasta el año 2005, efectuando una descripción del perfil epidemiológico de los pacientes que precisan TH, estudiando los códigos de inclusión en la lista de espera, las patologías que generaron dichas inclusiones, la compatibilidad isogrupo y la evolución pos-TH (necesidad de reTH y supervivencia posterior).

Durante el periodo de tiempo estudiado, se realizaron un total de 200 TH. El 60\% de los mismos fueron varones cuya media de edad fue de 54 años. Las principales causas por las que precisaron TH fueron la cirrosis enólica $70(35 \%)$, hepatitis vírica 37 $(18,5 \%)$, de origen mixto $38(19 \%)$, fulminante $6(3 \%)$, criptogénica $6(3 \%)$, hepatocarcinoma $4(2 \%)$, cirrosis biliar primaria 2 $(1 \%)$, autoinmune $1(0,5 \%)$, otras causas $10(5 \%) .26$ personas precisaron reTH (13\%). En el momento del TH, 78 pacientes se encontraban en en estadio C de Child (39\%). 183 TH se realizaron de modo programado $(91,5 \%), 10$ se hallaban en urgencia 0 (6 de ellos debido a un fracaso hepático agudo grave), y 7 en urgencia no 0 (urgencia de zona). Los pacientes permanecían en lista de espera una media de 38 días (0-248), que en el caso de urgencia 0 se redujo a 27 horas desde su inclusión. La compatibilidad isogrupo fue del $95 \%$, y el $5 \%$ no era compatible. La supervivencia de los pacientes es del $82 \%$ al año de realización del TH, del $75 \%$ a los 3 años y del $61 \%$ a los 6 años.

Los pacientes que requieren TH son varones, jóvenes, siendo la cirrosis enólica, la hepatitis vírica o de origen mixto las etiologías que más frecuentemente originan su indicación, al igual que en otros estudios en los que el VHC y el hepatocarcinoma son las principales indicaciones (2-4). El período de espera es reducido, sobre todo en el caso de encontrarse en urgencia 0 , ambos tiempos inferiores a las cifras del registro nacional de trasplantes, lo que posiblemente traduce una desigualdad en el acceso a estos programas como ya apuntan algunas investigaciones (3-5). Un elevado porcentaje de pacientes se encuentra en grado muy avanzado de la enfermedad, y una gran proporción de los mismos recibe un hígado de donante compatible. La necesidad de reTH es escasa, motivada fundamentalmente por fallo hepático fulminante al igual que en otros estudios $(6,7)$. La supervivencia al año, así como a los 3 años es elevada, y sobreviviendo más de la mitad de los pacientes una vez transcurridos 6 años. En la actualidad la supervivencia de los pacientes $\mathrm{TH}$ es superior a la del inicio de estos programas, probablemente por una mejoría de las técnicas quirúrgicas y de los cuidados posquirúrgicos en las unidades de cuidados intensivos, así como por el desarrollo de nuevos agentes inmunosupresores, y una mejor selección de los candidatos (2).

\section{B. Obón Azuara, I. Gutiérrez Cía, P. Luque Gómez}

Servicio de Medicina Intensiva. Hospital Clínico Universitario. Zaragoza

1. Starzi TE, Brettschneider L, Penn I, Giles G, Picache R, Putnam CW. Clinical liver transplantation. Transplant Rev 1969; 2: 3-68.

2. Amador A, Charco R, Marti J, Ferrer J, Mans E, et al. Mil trasplantes en el Hospital Clinic I Provincial de Barcelona. Cir Esp 2005; 78: 231-7.

3. Vargas V, Rimola A, Casanovas T, Castells L, Navasa M, Baliellas C, et al. Applicability of liver transplantation in Catalonia at the end of the millennium. A prospective study of adult patient selection for liver transplantation.Transpl Int 2003; 16: 270-5.

4. Cuende Melero N, Miranda Serrano B, Canon Campos JF, Naya Nieto T, Garrido Cantarero G. Criterios de priorización para el acceso al trasplante. El caso del trasplante hepático en España. Med Clin (Barc) 2003; 120: 380-6.

5. Sanchez-Bueno F, Cuende N, Matesanz R, Parrilla P. Emergency organ transplantation in Spain: liver emergency and outcomes. Transplant Proc 2005; 37: 3878-80.

6. Landaverde C, Berenguer M, Aguilera V, San Juan F, Prieto M, Berenguer J. Retrasplante hepático: análisis de los resultados en 50 pacientes. Med Clin (Barc) 2005; 124: 721-5.

7. García Gil FA, Luque P, Ridruejo R, Tome Zelaya E, Guemes A, Serrano MT, et al. Liver transplant, in emergency 0 (UNOS Status 1). Transplant Proc 2006; 38: 2465-7. 